\title{
Electromagnetic induction imaging with atomic magnetometers
}

\author{
Cameron Deans, Luca Marmugi, Ferruccio Renzoni \\ Department of Physics \& Astronomy, University College London, GowerStreet, London, UK, WC1E 6BT \\ cameron.deans.14@ucl.ac.uk
}

\begin{abstract}
We report on the development and performance of an electromagnetic induction imaging system based on atomic magnetometers. We discuss, and demonstrate experimentally, potential applications of our system in the security, industrial, and biomedical fields.
\end{abstract}

OCIS codes: $110.0110,280.4788$.

\section{Introduction}

Electromagnetic induction imaging, or magnetic induction tomography (MIT), is a technique capable of imaging the electric and magnetic properties of objects [1]. In MIT an oscillating magnetic field is applied from an excitation coil to induce eddy currents in the target. These eddy currents generate an additional magnetic field component. The detection of this field provides insight into the electric and magnetic properties of the object. In particular, MIT can provide a direct mapping of all three passive dielectric properties; conductivity $\sigma$, permittivity $\varepsilon_{r}$, and permeability $\mu_{r}$. Electromagnetic induction images of target objects can be obtained by taking spatially resolved MIT measurements. Tomographic measurements can be constructed by taking measurements at various excitation field frequencies, thus varying the penetration of the field into the target.

Classical MIT systems use an inductive pick-up coil, or set of coils, for magnetic field measurements. However, this choice places limitations on the ultimate performance of the system. Instead, our imaging system is based on atomic magnetometers (AMs) [2,3]. These devices offer extreme sensitivity over a wide frequency range, from $\mathrm{Hz}$ to tens of MHz. This allows simple tuning of the excitation field in MIT measurements and overcomes the poor lowfrequency sensitivity of pick-up coils. Furthermore, miniaturised AMs could offer increased imaging resolution and robust operation in unshielded environments provides flexibility for potential applications.

An MIT imaging system would find applications in security, industrial, and biomedical imaging. Security applications include: the detection of concealed bodies, cargo screening, and remote surveillance. Industrial applications include: the localisation of foreign bodies and the detection of defects in components. Alternatively, MIT is an attractive method of medical imaging as it is non-invasive and no direct contact with the patient is required. A biomedical MIT imaging system could aid the diagnosis and treatment of specific diseases and conditions where a change in conductivity plays, or is expected to play, an important role. Examples include the detection and diagnosis of cardiac arrhythmias and malignant tumours $[4,5]$.

\section{Methods}

In this work, we describe the development, and demonstrate the performance of our AM-based electromagnetic induction imaging system. The core of the device is a radio-frequency atomic magnetometer (RF-AM) comprising of a vapour cell of rubidium atoms interrogated by laser beams. The RF-AM measures the properties of an oscillating magnetic field produced by an excitation coil. When a target object is placed in the oscillating field eddy currents are induced in the object that modify the magnetic field's properties. The RF-AM records these changes allowing us to build up images of the object by performing position resolved measurements [3].

\section{Results}

Typical electromagnetic induction images produced by our system are shown in Figure 1. The scans consist of a range of materials and shapes (with a wide range in conductivity). We show that it is possible to image different parameters, such as amplitude R, phase shift $(\phi)$, and in-phase component X. Each of these equates to the mapping of different electromagnetic properties of the target object. Finally, these images demonstrate wide range of operation frequencies 

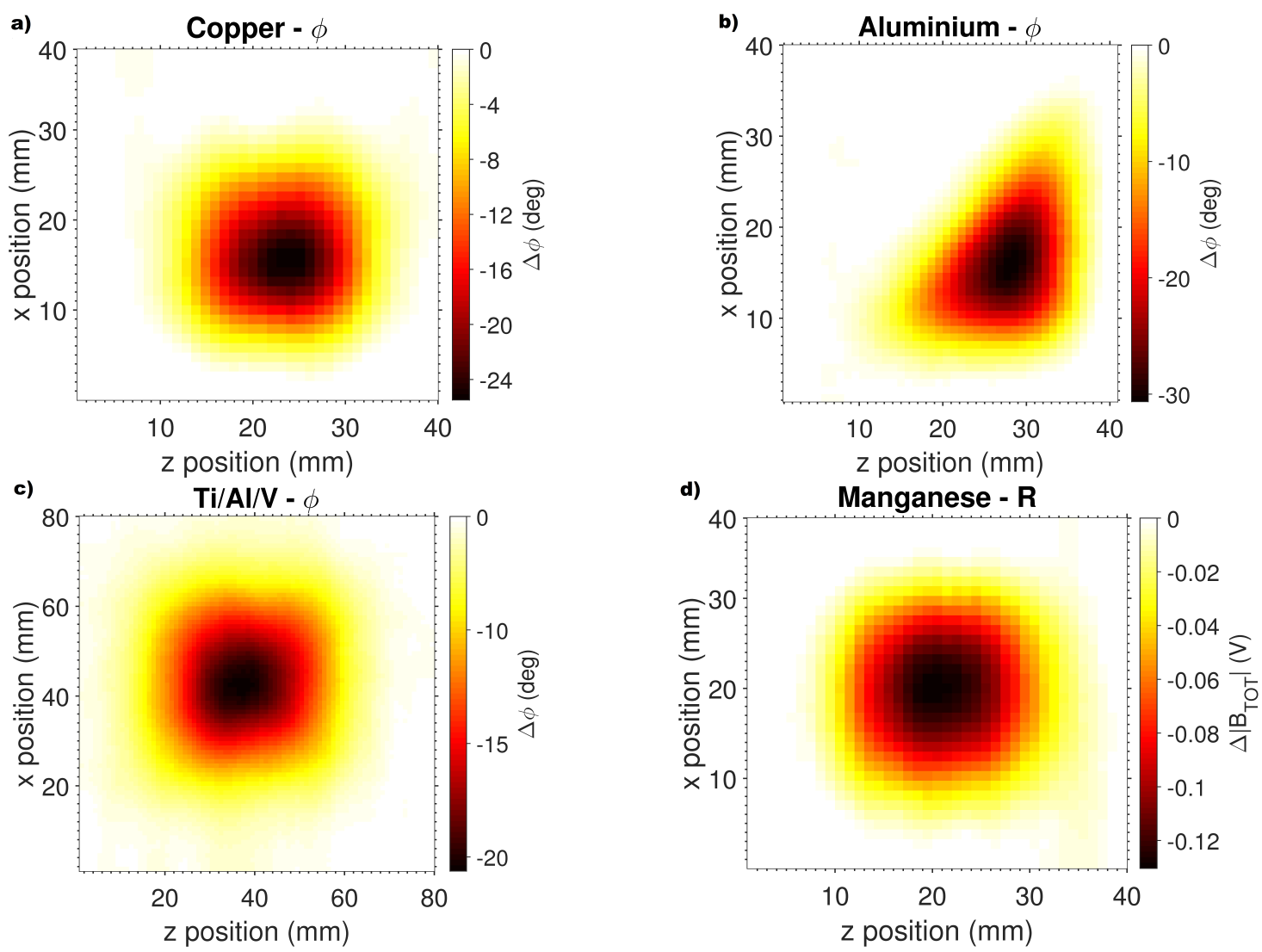

Fig. 1. Electromagnetic induction imaging with an AM: a) $\phi$ image of a Copper square at $1.89 \mathrm{kHz}\left(25 \mathrm{~mm} \times 25 \mathrm{~mm}, 1 \mathrm{~mm}\right.$ thick. $\left.\left.\sigma_{\mathrm{Cu}}=5.98 \times 10^{7} \mathrm{Sm}^{-1}\right), \mathbf{b}\right) \phi$ image of an Al triangle at $1 \mathrm{kHz}\left(31 \mathrm{~mm} \times 38 \mathrm{~mm} \times 50 \mathrm{~mm}, 3 \mathrm{~mm}\right.$ thick. $\left.\sigma_{\mathrm{Al}}=3.77 \times 10^{7} \mathrm{~S} \mathrm{~m}^{-1}\right)$, c) $\phi$ image of a Ti/Al/V square at $250 \mathrm{kHz}\left(50 \mathrm{~mm} \times 50 \mathrm{~mm}, 1 \mathrm{~mm}\right.$ thick. $\left.\sigma_{\mathrm{Ti} / \mathrm{Al} / \mathrm{V}}=5.95 \times 10^{5} \mathrm{~S} \mathrm{~m}^{-1}\right)$, d) Radius image of a Mn square at $208 \mathrm{kHz}\left(25 \mathrm{~mm} \times 25 \mathrm{~mm}, 1 \mathrm{~mm}\right.$ thick. $\left.\sigma_{\mathrm{Mn}}=5.4 \times 10^{5} \mathrm{~S} \mathrm{~m}^{-1}\right)$.

available and the ease with which the operating frequency is adapted based on the target material, penetration depth, or desired application.

Each image consists of a matrix of position-dependent measurements. For each image only a single scan is taken, with no background subtraction required. The data is collected point-by-point in real time. The images accurately reproduce the size and shape of the target objects, with no image reconstruction algorithm applied. The lack of sharpness at the corners is attributed to boundary effects, due to interruption of the circular flow of eddy currents in those regions.

In addition to different materials imaging, we demonstrate the potential applications of our imaging system with the following proof-of-principle experiments.

\subsection{Non-destructive evaluation}

Firstly, we consider non-destructive evaluation (NDE) of materials. An example of which is the detection of defects in critical components. We explored this application by investigating a small crack $(<1 \mathrm{~mm})$ in an aluminium ring. The crack (Figure 2a)) disrupts the flow of eddy currents. As a result there is a local decrease in the MIT response field in the region of the crack. This decrease is detected in both Radius and $\phi$ maps of the ring at $10 \mathrm{kHz}$ (Figures $2 \mathbf{b}$ ) and 2c)), respectively [3].

\subsection{Imaging through conductive barriers}

A second application of MIT imaging is the penetration of conductive barriers. This shows the potential for MIT imaging in security and screening applications. 

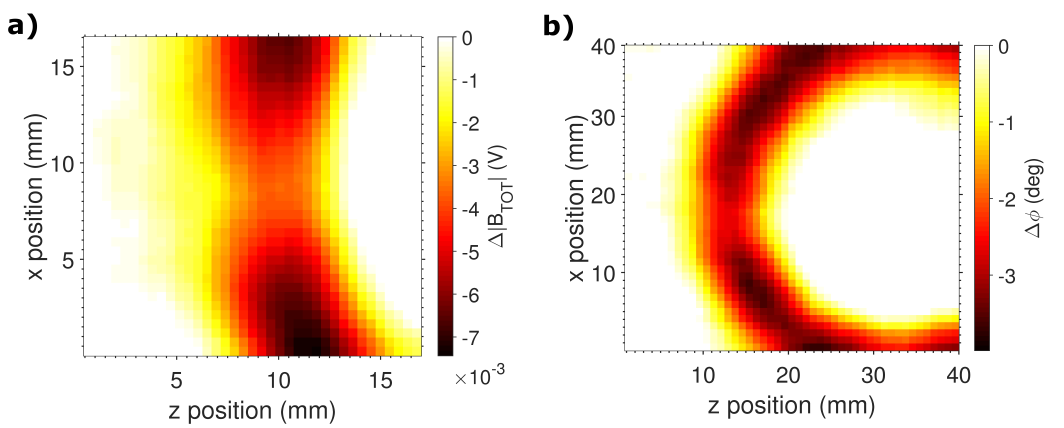

Fig. 2. Non-destructive evaluation: $6 \mathrm{~mm}$ cracked Al ring, $2 \mathrm{~mm}$ thick, crack $<1 \mathrm{~mm}$. Electromagnetic induction images at $10 \mathrm{kHz}$ : a) high-resolution radius image, b) $\phi$ image.

Figure 3 shows a copper square (side-length $25 \mathrm{~mm}, 1 \mathrm{~mm}$ thick) concealed behind a large aluminium barrier of thickness $1 \mathrm{~mm}$. Tuning the RF-OAM frequency to $500 \mathrm{~Hz}$ (to ensure penetration of the $\mathrm{Al}$ shield), the Cu square can be imaged in a single scan without any background subtraction or reconstruction techniques. The blurring at the edges of the $\mathrm{Cu}$ square can be attributed to the flow of eddy currents between them, as the objects are in electrical contact.

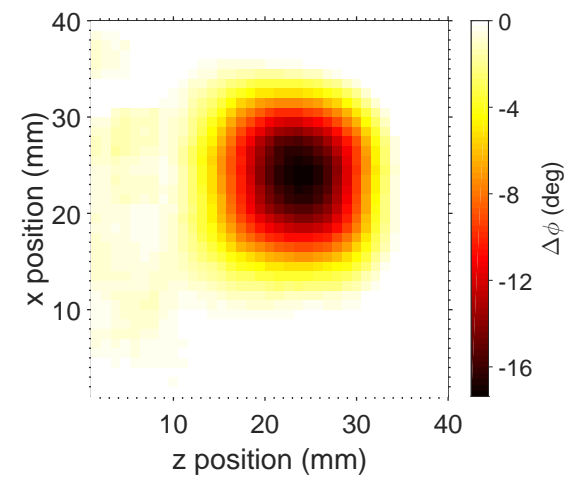

Fig. 3. Electromagnetic induction imaging through a conductive barrier: $\phi$ image of $\mathrm{Cu}$ square (side-length $25 \mathrm{~mm}, 1 \mathrm{~mm}$ thick) through a $1 \mathrm{~mm}$ thick Al barrier at $500 \mathrm{~Hz}$.

\section{Conclusion}

Our results demonstrate the feasibility for the development of an imaging system based on AMs for electromagnetic induction imaging applications. The system is shown to perform over a wide frequency range facilitating the imaging of targets with a wide range in conductivities and the penetration of barriers. A future imaging system, based on arrays of AMs performing MIT measurements, could find applications in non-destructive evaluation of materials, security and screening, and medical imaging.

\section{References}

1. H. Griffiths, "Magnetic induction tomography," Measurement science and technology 12, 1126 (2001).

2. A. Wickenbrock, S. Jurgilas, A. Dow, L. Marmugi, and F. Renzoni, "Magnetic induction tomography using an all-optical ${ }^{8} 7 \mathrm{Rb}$ atomic magnetometer," Optics Letters 39, 6367-6370 (2014).

3. C. Deans, L. Marmugi, S. Hussain, and F. Renzoni, "Electromagnetic induction imaging with a radio-frequency atomic magnetometer," Applied Physics Letters 108, 103,503 (2016).

4. L. Marmugi and F. Renzoni, "Optical magnetic induction tomography of the heart," Scientific reports 6 (2016).

5. C. Deans, L. Marmugi, S. Hussain, and F. Renzoni, "Optical atomic magnetometry for magnetic induction imaging of the heart," Proc. SPIE 9900 pp. 99,000F-99,000F (2016). 